\title{
Die Erfahrungen der Väter im Kreißsaal
}

\author{
Anne Köhne, Hebamme B.Sc., Prof. Dr. Claudia Hellmers, Osnabrück
}

In Deutschland werden $90 \%$ der Frauen von ihrem Partner bzw. vom werdenden Vater in den Kreißsaal begleitet [1]. Die Väter als Begleitpersonen während der Geburt sind Alltag im Kreißsaal. Trotzdem gibt es kaum Forschung über ihre Erfahrungen während der Geburt. Welche Sicht haben die Väter auf das Geburtsgeschehen? Welche Gefühle begleiten sie während des Geburtsprozesses? Die Autorin untersuchte diese Fragen in ihrer Bachelorarbeit im Studiengang Midwifery an der Hochschule Osnabrück.

\section{Hintergrund}

Mitte des letzten Jahrhunderts fand in Deutschland ein Wandel in der Geburtshilfe statt: Die Frauen gebären immer häufiger im Krankenhaus und immer seltener zuhause [2]. In den 1970er Jahren wurden die werdenden Väter zunehmend in die Geburtsbegleitung im Kreißsaal eingebunden [3]. Sie wurden in den letzten 30 Jahren als Unterstützer während der Geburt zur Normalität. Heutzutage begleitet die Mehrheit der Väter (über 90\%) ihre Partnerinnen zur Geburt $[1,4,5]$.

\section{Vorteile der Geburtsbegleitung}

Werdende Väter unterstützen ihre Frauen emotional und körperlich. Zudem agieren sie als Vermittler und Anwalt ihrer Partnerin in der Kommunikation mit dem Personal oder beim Treffen von Entscheidungen [6, 3]. Ihre Begleitung hat Vorteile für das peripartale Outcome. Die Gebärenden waren mit ihrem Geburtserlebnis zufriedener, sie haben weniger Schmerzen und sind seltener erschöpft. Infolgedessen ist die Interventionsrate reduziert: weniger Medikamenteneinsatz, eine geringere Rate an Periduralanästhesien sowie weniger instrumentale und operative Entbindungen. Die Anwesenheit des Vaters/Partners vertieft die Paarbeziehung und unterstützt die Vater-Kind-Bindung [7, 8, 5].

Mit der Einbindung der Väter in das Geburtsgeschehen kann der Geburtsprozess für die Frauen und für die gesamte Familie positiver gestaltet werden [6].

\section{Die Perspektive der Männer}

Es gibt nur wenige wissenschaftliche Arbeiten, die sich mit den Perspektiven von Männern beschäftigen, die ihre Partnerinnen während der Geburt begleitet haben. Eine sehr geringe Anzahl von Studien untersucht die Gefühle, das Erleben und die Bedürfnisse der Männer. Johansson et al. stellen fest, dass die Geburt von den Vätern als positives und aufregendes Erlebnis beschrieben wird. Die meisten Männer genießen das Geburtsgeschehen und berichten von angenehmen und erfreulichen Gefühlen [9]. Auch Chan und Paterson-Brown berichten von einer Bereicherung, die Väter durch die Geburt ihres Kindes erfahren [10]. Die Väter beschreiben die Geburt als einen lebensverändernden und überwältigenden Moment [11].
Die Gefühle und Bedürfnisse der Väter/ Partner erhalten eher selten Aufmerksamkeit im Kreißsaal. Man muss davon ausgehen, dass sich ihr Erleben vom Erleben der Frauen unterscheidet und deshalb auch ihre Bedürfnisse nicht mit denen der Frauen identisch sind [10, 4]. Eine differenziertere Untersuchung ihres Erlebens während der Geburt ist wichtig, damit die Väter/Partner bei der Geburtsbegleitung besser berücksichtigt werden können. Wenn die Rücksichtnahme auf die Väter erhöht wird, ist dies ein Mehrwert für die ganze Familie, insbesondere für die Frau. Es scheint notwendig, die väterliche Perspektive aufzudecken, um Hebammen und anderes medizinisches Personal zu sensibilisieren.

Bislang fehlen Forschungserkenntnisse zum Erleben der Väter/Partner aus Deutschland. Die bisherigen internationalen Forschungserkenntnisse sind noch unzureichend. Außerdem können diese nur sehr begrenzt auf Deutschland übertragen werden, aufgrund kultureller Unterschiede. Zudem bestehen zwischen den verschiedenen Gesundheitssystemen große Unterschiede.

\section{Eigene Untersuchung}

Gegenstand dieser Untersuchung sind die Erfahrungen der geburtsbegleitenden 
Tab. 1 Methodik der Untersuchung.

\begin{tabular}{|l|l|l|l|l|l|l|l}
\hline Methodik & qualitative Untersuchung zur Ermittlung subjektiver Daten \\
\hline Teilnehmer & $\begin{array}{l}12 \text { Väter im Alter von 26-44 Jahren, davon 11 nach Klinik- } \\
\text { geburt, einer nach Geburt in Geburtshaus }\end{array}$ \\
\hline Einschlusskriterien & $\begin{array}{l}\text { Väter, die ihre Partnerin zur Geburt begleitet haben und } \\
\text { deren neugeborenes Kind zum Zeitpunkt der Befragung } \\
\text { jünger als 17 Wochen alt ist }\end{array}$ \\
\hline Ausschlusskriterium & $\begin{array}{l}\text { Geburt des Kindes mit primärem oder geplantem Kaiser- } \\
\text { schnitt }\end{array}$ \\
\hline Feldzugang & $\begin{array}{l}\text { Gewinnung der Studienteilnehmer über Hebammen und } \\
\text { Hebammenpraxen in Niedersachsen und im Großraum } \\
\text { Hamburg }\end{array}$ \\
\hline Datenerhebung & $\begin{array}{l}\text { retrospektive leitfadengestützte Einzelinterviews im } \\
\text { September und Oktober 2013 }\end{array}$ \\
\hline Auswertung & qualitative Inhaltsanalyse nach Mayring [12] \\
\hline Ethische Kriterien & $\begin{array}{l}\text { Information über das Forschungsvorhaben, } \\
\text { freiwillige Teilnahme, Anonymisierung der Daten }\end{array}$ \\
\hline
\end{tabular}

Väter. Folgende Fragen leiten die Untersuchung:

- Wie schildern Männer ihre Rolle während der Geburtsbegleitung ihrer Partnerin?

- Was erleben Männer, die ihre Partnerin während der Geburt begleiten?

- Welche Wünsche und Bedürfnisse haben Männer, die ihre Partnerin während der Geburt begleiten?

\section{Ergebnisse}

\section{Die Rolle der Väter/Partner}

Die werdenden Väter und Partner der Frauen beschreiben bereits ihre Anwesenheit und ihre gemeinsamen Gespräche als eine Unterstützung für ihre Partnerin. Die Väter leisten damit emotionale Hilfe. Ein Studienteilnehmer schildert das folgendermaßen:

„Indem ich einfach da war, sagte sie mir im Nachhinein: ,Einfach nur Dasein, [dich] dafür interessieren.' Ihr Mut zuzusprechen. Das war ihr wichtig." (TN 5, 162163).
Werdende Väter übernehmen Aufgaben bei der Geburtsbegleitung und dienen damit häufig der körperlichen Unterstützung ihrer Partnerin. Die Interviewteilnehmer haben folgende Aufgaben übernommen: Unterstützung beim Umhergehen und Bewegen, beim Ausprobieren von Geburtspositionen, körperliche Zuwendung und Massieren, Getränke und Essen bringen, zur Atmung anleiten. Ein Vater schildert sein Tun während der Geburtsbegleitung sehr anschaulich:

„Einfach durch zureden, durch Wasser bringen, Wasser besorgen, Musik anmachen, beim Umdrehen helfen, Decke drüber, Decke wieder weg, Decke wieder drüber, warm, kalt, Hände zerquetschen lassen, (...) immer wieder zu den Hebammen gehen - ,Ihr müsst noch mal gucken!‘ - Dieses ständige Dasein, Helfen, Unterstützen, Machen, Tun, Licht an, Licht aus.“ (TN 7, 163-167).

Die Männer vermitteln zwischen ihrer Partnerin und dem geburtshilflichen Personal und übernehmen damit die informationelle Unterstützung und geben ihrer Partnerin so Sicherheit. Ein Studi- enteilnehmer beschreibt seine Rolle sehr klar:

„Aber wenn man da ankommt - die meisten Menschen, die da sind, sind alle Fremde. Man kennt diese Leute nicht. Ich kenn diese Person nicht, wie soll ich dieser Person jetzt vertrauen? Deswegen finde ich, dass der Mann in dem Fall eine wichtige Rolle hat. Weil ich bin jemand, den sie kennt und sie weiß genau oder kann bei meinem Gesichtsausdruck auch wissen, ob ich lüge oder nicht. Wenn ich sage: ,Es ist alles in Ordnung!' Dann merkt sie sofort, das ist nicht in Ordnung. [...] Deswegen gibt man auch ein bisschen mehr sicheres Gefühl. Das ist auch die Rolle vom Mann. Wir in unserer Rolle.“(TN 10, 246-253).

Den befragten Vätern ist zudem bewusst, dass sie nicht die Hauptpersonen sind und sie versuchen deshalb das Personal nicht zu stören.

„Aber man weiß es auch, (...) da ist man das fünfte Rad am Wagen, ganz klar. Das habe ich jetzt auch niemandem übel genommen. Das war vollkommen ok für mich, dass ich mich in dieser Rolle jetzt wiedergefunden habe (...).“ (TN 5, 104108).

„Ich habe versucht, nicht im Weg zu stehen." (TN 3, 249-250).

\section{Die Erfahrungen der Väter/Partner}

Für die Väter sind die Wehenschmerzen ihrer Partnerinnen nicht greifbar. Diese Unfähigkeit, die Schmerzen nachzuempfinden und ihre Partnerin zu entlasten, löst bei ihnen Gefühle der Hilflosigkeit aus. Die Väter beschreiben, dass sie ihren Partnerinnen bei der Bewältigung der Wehenschmerzen nicht helfen können und sie sich deshalb hilflos fühlen:

„Ich habe schon ein paar Mal gedacht, was kann ich machen? [Ich] bin dann zum Schluss gekommen, ich kann nichts machen.“(TN 3, 96-97). 
„Man ist relativ hilflos und auch relativ verzweifelt, weil es bei ihr auch relativ heftig war. Man muss dann einfach irgendwie durch.“(TN 7, 81-82).

Zudem hemmen die Abläufe im Kreißsaal die Väter/Partner bei der Unterstützung ihrer Partnerinnen. So führt der Wunsch, für die Partnerin da zu sein und gleichzeitig der Hebamme und dem medizinischen Personal nicht im Wege zu stehen, zu einem inneren Konflikt. Dies macht die Väter/Partner oft hilflos:

„Na klar ist man noch ein Stückweit hilflos, aber das ist man nur durch die Arbeitsschritte dort und wenn man gerade nicht weiß wohin mit den Händen."(TN1, 153-155).

Einige Studienteilnehmer schildern die Diskrepanz zwischen ihren Erwartungen und der tatsächlichen Dauer der Geburt:

„Also die Zeit, das ist, was ich nicht erwartet habe. Gerade bei unserem Sohn, war es natürlich extrem. Die Zeit! Dass Schmerzen kommen, war mir klar. Dann aber auch wieder über die Dauer. Das fand ich schon heftig. Das hat mich überrascht [...].“ (TN 3, 185-189).

„Ich dachte, jetzt fängt es langsam an. Und als ich dachte es fängt an, da war das Kind da." (TN 1, 65-66).

Die Geburt des Kindes ist für die Väter ein sehr bewegender Moment. Sie schildern sehr positive Gefühle und wie ihre Anspannung von ihnen abfällt.

„[...] Als sie dann draußen war, war es so eine komplette Lösung von sämtlicher Anspannung, was sich darin geäußert hat, dass mir dann doch längerfristig die Tränen kamen." (TN 2, 226-228).

Die befragten Väter beschreiben ihr Erleben von Interventionen während der Geburt als Stress und Nervosität. Sie machen sich Sorgen um ihre Frau und ihr Kind. Ebenso haben das Verhalten und die Kommunikation des Personals bedeutsame Auswirkungen auf die Väter und ihre Gefühle.
„Was mich nervös gemacht hatte, war als die Hebamme sehr darauf pochte, den Wehenschreiber anzuschließen. Mit dieser Begründung: ,Wenn ich das jetzt nicht mache und das Kind hat dann irgendwie Sauerstoffmangel unter der Geburt.' (137140) [...] Das macht mir eher Angst, als dass es mich beruhigt, wenn da ein Gerät angeschlossen ist." (TN 2, 172-173).

„Aber wenn jemand sagt: ,Oh, oh!' oder "Jetzt müssen wir wirklich etwas tun!" Dann hast du sofort Angst. Weil man hört auch viele Geschichten. Leider ist das so. Man will hoffen, dass nichts Schlimmes passiert. [...] wenn man sagt: ,Der Herzschlag ist runter gekommen!' [...] Dein Kopf fängt an zu spinnen mit Gedanken, die im Moment nicht wirklich gut passen." (TN 10, 158-163).

„[Als] die Geburt richtig losging, war es richtig grausam. [...] Der Arzt hat sich auf [...] [meine Frau] raufgelegt und bei jeder Wehe mitgedrückt. Den Ellenbogen in den Bauch reingedrückt. Ich wusste überhaupt nicht, was da passiert. Es sah einfach nur grausam aus. [...] Ich wäre am liebsten rausgegangen. Mein persönlicher Instinkt war Flüchten, weg hier, aus der Situation raus. [...] Das war schrecklich." (TN 12, 54-64).

\section{Bedürfnisse der Väter/Partner}

Die Väter/Partner wünschen sich, dass es ihrer Partnerin und ihrem Kind gut geht. Für eine nächste Geburt äußern sie nur wenige Wünsche.

„Da ist das erste, dass Frau und Kind gut drauf sind und dass es alles gut klappt. Bei der zukünftigen Geburt würde ich mir wünschen, dass es vielleicht ein bisschen schneller voran geht. Dass es dementsprechend nicht so die Erschöpfungsanfälle gibt.“(TN 9, 273-277).

„Da bin ich ganz ehrlich. Ich glaube, viel anders, viel, viel anders oder viel besser kann das eigentlich nicht sein." (TN 6, 253-254).
Die Interviewteilnehmer schildern die Ruhe und Sicherheit, die das geburtshilfliche Personal ausstrahlte und die beruhigende Wirkung.

„Ich habe noch nie Menschen erlebt, die in - für mich als werdender Vater in einer Stresssituation - so ruhig und besonnen geblieben sind. Das fand ich unheimlich bewundernswert, muss ich ganz ehrlich sagen. Das finde ich auch eine ganz große Leistung von den Frauen, die da arbeiten. Dass sie wirklich so viel Ruhe auch ausstrahlen, dass sie gleichzeitig nicht nur meine Frau, sondern auch mich unheimlich beruhigt haben. Da hatte ich zu keinem Zeitpunkt irgendwie das Gefühl alleingelassen $z u$ werden oder Angst zu haben oder wie auch immer - kein Stück." (TN 11, 222-229).

„Es war eine Hebamme mit im Raum und eine Ärztin. Die strahlen schon ziemlich viel Sicherheit aus. Ich meine, das ist ja auch wahrscheinlich die Aufgabe der Hebammen da. Aufjeden Fall Sicherheit hinüberzubringen. Das hat eigentlich gut funktioniert.“(TN 8, 95-98).

Die werdenden Väter wünschen sich gezielte Informationen und eine angemessene Aufklärung über das Vorgehen und notwendige Maßnahmen während der Geburt. Sie wollen nicht im Unklaren gelassen werden.

„ICh wusste zu jeder Zeit ganz gut, was sie gemacht haben. [...] Jeden Handgriff, den die Hebammen gemacht haben, haben sie uns erklärt und auch erzählt, warum sie es machen. Nicht einfach getan. Auch keinen besorgten Gesichtsausdruck gemacht oder wie auch immer. [...] Das fand ich auch ganz toll, dass man dann nicht im Unklaren gelassen wird." (TN 11, 240-247).

\section{Diskussion}

Viele der befragten Väter haben das Gefühl, während des Geburtsgeschehens im Weg zu stehen und sie wollen nicht stören. Auch Chandler und Field [13] stellten fest, dass Väter das Gefühl ver- 
mittelt bekommen, toleriert zu werden, aber dennoch nutzlos zu sein. Vehviläinen-Julkunen und Liukkonen empfehlen dem geburtshilflichen Personal, die Fragen und Meinungen der Väter zu hören [14].

Obwohl die Begleitung der Frauen durch ihre Partner in den Kreißsälen alltäglich geworden ist, ist die Rolle der werdenden Väter während der Geburt immer noch unklar. Es fehlen eindeutige Vorstellungen über die Aufgaben der begleitenden Väter. Es wird ihnen überlassen, sich ihre Rolle während der Geburt zu suchen [3]. Väter wünschen sich eine Aufgabe und wollen für ihre Partnerin eine Hilfe und Stütze sein. Gleichzeitig wollen sie die Arbeitsabläufe des geburtshilflichen Personals nicht stören. Dieser innerliche Konflikt führt zu der von den Vätern erlebten Hilflosigkeit.

\section{Besonders wichtig ist die Kommunika-} tion vor der Durchführung notwendiger Maßnahmen, da Väter ohne einleitende Aufklärung die Intervention nicht einordnen können und dies dann als Stress erleben.

Johansson und Hildingsson zeigen auf, dass die Art und Weise der Kommunikation während der Geburt Einfluss auf das Geburtserleben der Väter hat [8]. Väter wollen über die Maßnahmen und Geschehnisse während der Geburt informiert und aufgeklärt werden. Ein Mangel an Informationen führt bei Vätern zu Stress und in der Folge zu Unzufriedenheit mit dem Geburtserlebnis [9].

Die vom Personal ausgestrahlte Ruhe und Sicherheit wirkt sich beruhigend auf die geburtsbegleitenden Väter aus. Unzufriedenheit mit dem geburtshilflichen Personal kann zu negativen Geburtserlebnissen der Väter führen [9].

\section{Empfehlung}

Die Väter sind involviert und emotional beteiligt am Geburtsprozess. Wenn die gebärende Frau möchte, dass ihr Partner einbezogen wird, gehört die Betreuung des Paares zu den Aufgaben von Hebammen [15]. Hebammen sollten die Väter in ihre Betreuung einbeziehen und sich für die Perspektive der begleitenden Männer sensibilisieren. Die Unterstützung der Väter durch einen respektvollen Umgang und eine ehrliche Kommunikation sind ein wichtiger Beitrag für eine erfolgreiche Betreuung während der Geburt. Zudem wirkt sich das gemeinsame Geburtserlebnis positiv auf die Paarbeziehung aus.

Das Geburtserleben von Vätern/Partnern sollte in Geburtsvorbereitungskursen und Beratungsgesprächen während der Schwangerschaft thematisiert werden, damit die Paare die Möglichkeit haben, sich frühzeitig damit auseinanderzusetzen.

Frauen werden nicht nur vom Partner/ Kindsvater in den Kreißsaal begleitet, sondern auch von ihren Müttern, Schwestern, Freundinnen oder Partnerinnen. Deshalb sollte das Thema „Begleitpersonen der Gebärenden im Kreißsaal“ allgemein eine größere Bedeutung erlangen.

\section{Literatur}

1 BZgA (Bundeszentrale für gesundheitliche Aufklärung) (Hrsg.) (2012). Ich bin dabei! Vater werden. Köln

2 Draper J (1997). Whose welfare in the labour room? A discussion of the increasing trend of fathers' birth attendance. Midwifery, 13: 132-138

3 Wöckel A, Abou-Dakn M (2009). Väter im Kreißsaal - hilfreich oder überflüssig? Hebammenforum, 8: 623-627

4 David M, Aslan G, Siedentopf J-P, Kentenich H (2009). Ethnic Turkish fathers in birth support roles in a Berlin labour and delivery room - motives, preparation und incidence in a 10-year comparison. Journal of Psychosomatic Obstetrics and Gyecology, 30 (1): 5-10

5 Steen M, Downe S, Bamford N, Edozien L (2012). Not-patient and not-visitor: A metasythesis fathers' encounters with pregnancy, birth and maternity care. Midwifery, 28: 422-431
6 Enkin M, Keirse M, Neilson J, Crowther C, Duley L, Hodnett E, Hofmeyer J (2007). A guide to effective care in pregnancy and childbirth. 3rd Edition. Oxford: Oxford University Press

7 Brandao S, Figueiredo B (2012). Fathers' emotional involvement with the neonate: impact of the umbilical cord cutting experience. Journal of Advanced Nursing, 68 (12): 2730-2739

8 Johansson M, Hildingsson I (2013). Intrapartum care could be improved according to Swedish fathers: Mode of birth matters for satisfaction. Women and Birth, 26 (3): 195-201

9 Johansson M, Rubertsson C, Radestad I, Hildingsson I (2012). Childbirth - An emotional demanding experience for fathers. Sexual and Reproductive Healthcare, 3: 11-20

10 Chan K, Paterson-Brown S (2002). How do fathers feel after accompanying their partners in labour and delivery? Journal of Obstetrics and Gynaecology, 22 (1): 11-15

11 Erlandsson K, Lindgren H (2009). From belonging to belonging through a blessed moment of love for a child - the birth of a child from the fathers' perspective. Journal of Men's Health, 6 (4): 338-344

12 Mayring P (2010). Qualitative Inhaltsanalyse. 11. aktualisierte, überarbeitete Auflage. Weinheim: Beltz Verlag

13 Chandler S, Field P (1997). First-Time Fathers' Experience of Labor and Delivery. Journal of Nurse-Midwifery, 42 (1): 17-24

14 Vehviläinen-Julkunen K, Liukkonen A (1998). Fathers' experiences of childbirth. Midwifery, 14: 10-17

15 ICM (International Confederation of Midwives) (2013). Essential Competencies for basic Midwifery Practice. http://www. internationalmidwives.org (5.11.2013)

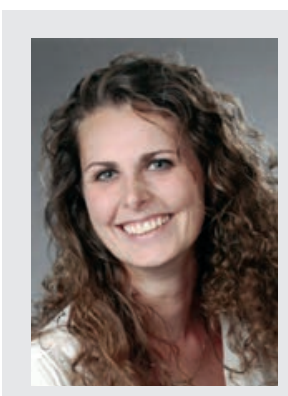

Anne Köhne

Hebamme B.Sc.

Anne.koehne@gmx.de 